\title{
From Live Cells to Caenorhabditis elegans: Selective Staining and Quantification of Lipid Structures Using a Fluorescent Hybrid Benzothiadiazole Derivative
}

\author{
Alberto A. R. Mota, ${ }^{\dagger}$ Jose R. Correa, $^{\dagger}$ Lorena P. de Andrade, $^{\dagger}$ José A. F. Assumpção, $^{\dagger}$
} Giovana A. de Souza Cintra, ${ }^{\ddagger}$ Lucio H. Freitas-Junior, ${ }^{\ddagger}$ Wender A. da Silva, ${ }^{\dagger}$ Heibbe C. B. de Oliveira, and Brenno A. D. Neto*,†(1)

\begin{abstract}
${ }^{\dagger}$ Laboratory of Medicinal and Technological Chemistry, University of Brasília (IQ-UnB), Campus Universitário Darcy Ribeiro, P.O. Box 4478, Brasília-DF CEP 70904970, Brazil

${ }^{\ddagger}$ Departamento de Microbiologia, Instituto de Ciências Biomédicas, Universidade de São Paulo (USP), 05508-900 São Paulo, São Paulo, Brasil
\end{abstract}

\section{Supporting Information}

ABSTRACT: The current article describes the synthesis, characterization, and application of a designed hybrid fluorescent BTD-coumarin (2,1,3-benzothiadiazole-coumarin) derivative (named BTD-Lip). The use of BTD-Lip for live-cells staining showed excellent results, and lipid droplets (LDs) could be selectively stained. When compared with the commercially available dye (BODIPY) for LD staining, it was noted that the designed hybrid fluorescence was capable of staining a considerable larger number of LDs in both live and fixed cells (ca. $40 \%$ more). The new dye was also tested on live Caenorhabditis elegans (complex model) and showed an impressive selectivity inside the worm, whereas the commercial dye showed no selectivity in the complex model.

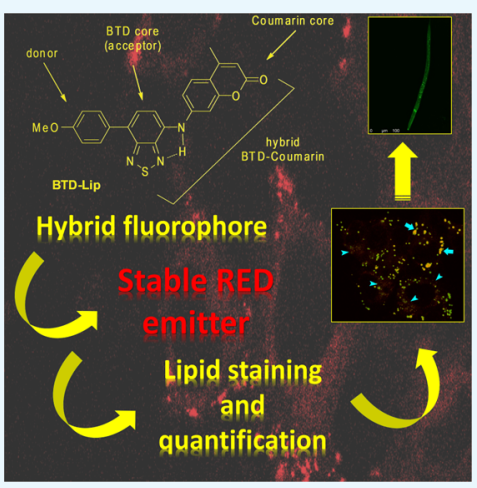

\section{INTRODUCTION}

Selective and stable small-molecule fluorophore development is of paramount importance for furthering diagnostics, chemical biology, medicine, and many other life sciences applications. ${ }^{1}$ Stability for long periods with no photobleaching is among highly desirable features for new fluorescent dyes. The challenge of developing new fluorescent compounds for cell imaging begins with the molecule design, meaning the new structure must be capable of transposing the cellular membrane and selectively stain a specific cell component or organelle inside the highly complex cellular environment. ${ }^{2}$ Afterward, stable and bright emissions are also mandatory for a precise subcellular localization and for dynamics studies. ${ }^{3}$ Commercially available dyes typically show fast photobleaching and have low chemical- and photostabilities.

Lipid droplets (LDs) are pivotal structures for life maintenance. ${ }^{4}$ BODIPY and Nile Red are the commonest commercially available fluorophores used for LD staining inside live cells, although their limitations such as leakage, photobleaching, blinking, low stabilities, and fade-off are very wellknown drawbacks, ${ }^{5}$ as very recently reviewed. ${ }^{6}$ On balance, only some LDs are stained using these two fluorophores, therefore limiting many studies and escaping both the distribution and the dynamics of other lipid types ${ }^{7}$ such as glycerolipids, grycerophospholipids, sphingolipids, and others. ${ }^{8}$
In other words, a large number of LD structures are not stained using the commercial dyes. LDs are known to be essential for energy supply and are associated with many health issues from obesity to neurodegenerative diseases as a straight consequence of lipid metabolism deregulation. The fast dynamics associated with LD (and lipid structures) ${ }^{9}$ is another drawback to monitor their live flux, and many types of lipid-based structures ${ }^{8}$ usually escape detection therefore turning LD detection and quantification an even more challengeable task. Another drawback of such a limitation is that misleading conclusions may be reached because many lipid structures escape from the detection when commercial dyes are used. In this sense, to the best of our knowledge, there is no lipid marker (fluorescent small molecules) capable of staining a large number of lipid types to follow their dynamics and to quantify the formed structures.

From cells to complex organisms, the gap and the challenges are even higher. The complexity increase has, as a consequence, the need for more selective and stable fluorophores capable of precise responses to fulfill the gap among these two astonishing worlds. Hence, the observed results inside live cells cultures will

Received: March 7, 2018

Accepted: March 26, 2018

Published: April 5, 2018 
not necessarily return similar results when model organisms such as Caenorhabditis elegans are used. Caenorhabditis elegans has been regarded as a platform for integrating chemical and biological research studies ${ }^{10}$ because of its attractive features such as (i) it is a multicellular organism (a nematode worm), (ii) it is transparent and facilitates fluorescence detection, (iii) it has organs and differentiated tissues, and (iv) $50-80 \%$ of its genes are homologues to humans. ${ }^{10}$ The use of such model organisms for lipid detection studies are fundamental, especially considering that lipid storages are known to be physiologically regulated therefore requiring intact animals ${ }^{11}$ for precise evaluations. Nearly 400 genes have been identified in C. elegans related to lipid storage, and these genes proved to have human homologues. ${ }^{12}$ At this point, one could fairly conclude that only cellular studies of lipids are not enough for a deep comprehension on the actual roles and consequences of lipid deregulations.

To come through these limitations, few fluorogenic smallmolecule dyes have been developed in recent years trying to surpass such boundaries and aiming at specific lipid staining. ${ }^{7,13-16}$ Some of these small molecule dyes were indeed tested and compared against C. elegans, ${ }^{13,17,18}$ but displaying severe limitations such as lack of sensitivity or lack of selectivity in the living nematode. Those new fluorophores likewise require several synthetic steps, showed some leakage, and stained most lipids observed with commercial dyes, typically LD. Bearing in mind that live cells and the worm C. elegans also have other lipid types, ${ }^{19}$ these lipids will likely escape detection when such dyes are used.

2,1,3-Benzothiadiazole (BTD) fluorophores are a new class of stable emitters capable of transposing the cell membrane and with high chemical- and photostabilities, as we have recently highlighted. $^{20}$ By using BTD-based fluorophores, some interesting applications were described. For instance, tumor cells under hypoxic condition were labeled, ${ }^{21}$ ligand trafficking in artificial and living cell systems could be probed, ${ }^{22}$ and other applications are found. ${ }^{23-27}$ Among these new applications, selective LDs staining was described by us ${ }^{28}$ and by others. ${ }^{29}$ We have shown the potential of BTD fluorophores for selective mitochondrial staining, ${ }^{30}$ for dynamic and endocytic internalization studies, ${ }^{28}$ and others, ${ }^{31,32}$ as we have recently highlighted. $^{20}$ In this perspective, we envisaged the synthesis of a fluorescent BTD-coumarin hybrid (named BTD-Lip) aiming at a stable lipid probe for live cell imaging and to test it in the complexity of the nematode C. elegans for lipid detection and quantification. Herein, we disclose our findings.

\section{RESULTS AND DISCUSSION}

Scheme 1 shows the synthesis of the new BTD-coumarin fluorescent hybrid compound. The designed structure should, in principle, overcome the aforementioned drawbacks because of the highly desirable chemical- and photophysical properties of fluorescent BTD derivatives, as reviewed elsewhere, ${ }^{33,34}$ therefore improving the beneficial photophysical properties of lipophilic coumarin derivatives. ${ }^{17}$ The use of hybrid derivatives is indeed an elegant strategy gaining attention in recent years. ${ }^{35}$

For the new BTD hybrid structure, we used a $p$ methoxyphenyl group on one side of the BTD core aiming at taking advantage of its electron donation property. BTD is known to be an efficient electron acceptor, ${ }^{33}$ and therefore this push-pull arrangement may facilitate the intramolecular charge transfer (ICT) in the excited state. On the other side, a coumarin group was attached in accordance with the strategy of
Scheme 1. Synthesis of the Fluorescent BTD-Coumarin Hybrid (Named BTD-Lip) ${ }^{a}$

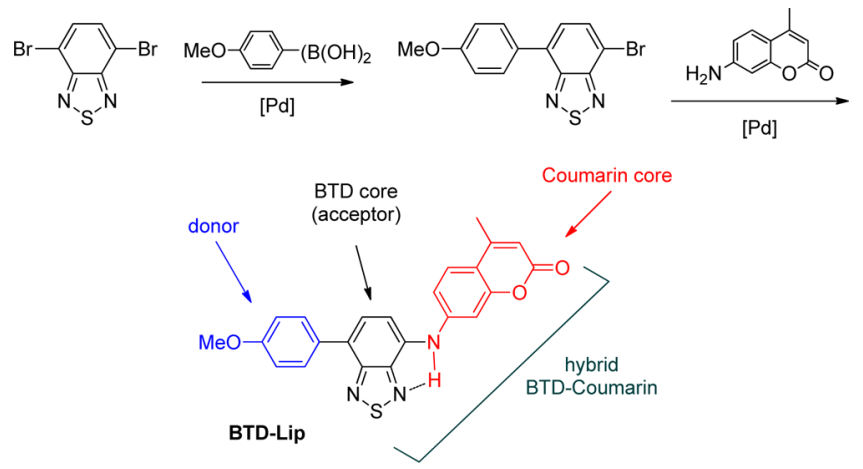

${ }^{a}$ Note the structure was designed to have improved photophysical properties because of the donor-acceptor molecular architecture and to improve the stability of the lipophilic coumarin moiety.

hybrid fluorophores, as explained before. Although several lipophilic groups could be used to tune this feature, the coumarin core would also extend the $\pi$-conjugation and help in the ICT process. The coumarin scaffold has a lipophilic character $^{36}$ and is capable of undergoing an efficient ICT with the adequate substituent, as recently demonstrated by Rendina and co-workers. ${ }^{36}$ In the current work, the coumarin is attached in the BTD extending the $\pi$-conjugation and substituted at its C7-position (coumarin as reference), that is, a position known to facilitate a light-up effect and ICT of fluorescent coumarin derivatives. $^{36}$ Tian and co-workers ${ }^{37}$ also used the lipophilic character of a C7-substituted coumarin to design a structure to intracellular stain of liposomal structures. In another example, Raić-Malić and co-workers ${ }^{38}$ linked triazole groups specifically at the C7-position of the coumarin core to improve its physicochemical properties. With the benefit of the available knowledge, we could therefore expect the new designed structure BTD-Lip to display improved lipophilic character with improved photo- and physicochemical properties.

As depicted from Scheme 1, the hybrid compound BTD-Lip was obtained in two steps and had its photophysical properties evaluated. Table 1 summarizes the obtained results and Figure S1 (in the Supporting Information file) shows the UV-vis, fluorescence emission, and Stokes shift $\left(\mathrm{cm}^{-1}\right)$ versus $E_{\mathrm{T}}^{\mathrm{N}}$ values for the tested solvents (solvatochromic method ${ }^{39,40}$ ) plots of

Table 1. Photophysical Data (in Different Solvents) for BTD-Lip (10 $\mu \mathrm{M}$ Solutions for All Analyses)

\begin{tabular}{lcccc}
\multicolumn{1}{c}{ solvents } & $\begin{array}{c}\lambda_{\max }(\mathrm{abs}) \\
(\mathrm{nm})\end{array}$ & $\log \varepsilon$ & $\begin{array}{c}\lambda_{\max }(\mathrm{em}) \\
(\mathrm{nm})\end{array}$ & $\begin{array}{c}\text { Stokes shift } \\
(\mathrm{nm})\end{array}$ \\
hexane & 467 & 3.42 & 585 & 118 \\
toluene & 460 & 3.96 & 600 & 140 \\
ethyl acetate & 465 & 3.79 & 605 & 140 \\
water & 483 & 3.64 & 628 & 145 \\
dichloromethane $^{a}$ & 462 & 3.91 & 608 & 146 \\
chloroform $^{\text {ethanol }}$ & 459 & 3.81 & 605 & 146 \\
methanol $_{\text {acetonitrile }}{ }^{a}$ & 463 & 3.96 & 626 & 163 \\
DMSO & 459 & 3.96 & 640 & 181 \\
& 455 & 3.88 & 624 & 169
\end{tabular}

${ }^{a}$ Quantum yield of fluorescence: $0.20(\mathrm{MeCN}), 0.25\left(\mathrm{CH}_{2} \mathrm{Cl}_{2}\right), 0.23$ $\left(\mathrm{CHCl}_{3}\right), 0.37(\mathrm{PhMe}), 0.01\left(\mathrm{H}_{2} \mathrm{O}\right)$, and $0.09(\mathrm{EtOH})$. Quinine sulfate in $1 \mathrm{M} \mathrm{H}_{2} \mathrm{SO}_{4}\left(\phi_{\mathrm{f}}=0.55\right)$, as standard. 
the new fluorescent BTD-Lip. Overall, results show the high stability of the designed compound, as indicated by its large values of Stoke shifts $(118-181 \mathrm{~nm})$ and molar extinction coefficients $(\log \varepsilon 3.42-3.96)$. The large Stoke shifts are likely a consequence of the efficient ICT, as noted for coumarin derivatives bearing this feature. ${ }^{41}$ This effect is also a consequence of the push-pull architecture ${ }^{42}$ of the designed structure. The efficient push-pull observed in the BTD-Lip structure facilitates the ICT, and therefore large Stokes shift may be noted in such cases. ${ }^{43}$

An evaluation of the probe using an aqueous soluble lipophilic environment, that is, using sodium dodecyl sulfate below and above the critical micellar concentration (CMC, 8 $\left.\mathrm{mmol} \mathrm{L}^{-1}\right)^{44}$ was also performed (Figure S5). As depicted from Figure S5, the fluorescence intensity increases (light up) after the CMC is reached, indicating an affinity for lipophilic regions as will be discussed and showed in the cellular experiments.

To be sure about the low cytotoxicity of BTD-Lip, 3-[4,5dimethylthiazol-2-yl]-2,5-diphenyltetrazolium bromide (MTT) experiments were performed and showed that the hybrid BTD fluorescent derivative is indeed noncytotoxic for the cells (Figure S2). Afterward, BTD-Lip also had its capacity as a selective cell marker initially tested against Caco- 2 cancer cell lineage (Figure 1).

The designed dye was tested using a considerably lessconcentrated solution $(3 \mu \mathrm{M})$ when compared with the commercial dye BODIPY $(12 \mu \mathrm{M})$ used to stain LD (see the Experimental Section in the Supporting Information file for details).

To confirm the precise subcellular localization of BTD-Lip and to confirm the selective LD staining, an additional assay was performed using the LD marker BODIPY, a widely commercially available dye used to stain lipids. The analyses show that the spherical organelles stained by BTD-Lip are in fact $\mathrm{LD}$ (Figure 1). The fluorescent pattern and distribution obtained using the commercial BODIPY (in both live and fixed cells) proved to be close to that with BTD-Lip. The fluorescent overlay images (Figure $1 \mathrm{C}, \mathrm{G}$ ) showed a yellow emission, that is, a perfect match of the combination of the green and red emissions using BODIPY and BTD-Lip. The distribution of the LD stained with BTD-Lip is however slightly different from those using BODIPY. BTD-Lip clearly stained considerably more spherical structures than those observed by using BODIPY. This feature shows that BTD-Lip has the affinity to other lipid structures rather than those stained by the commercial dye, a known limitation when BODIPY or Nile Red is used. ${ }^{6}$ Looking at the dots in yellow color (Figure 1), one may see that their number and distribution are very similar to that of BODIPY staining pattern. Looking at the dots in red color however one sees that these dots are much more distributed and in higher number than those observed in yellow color. This difference indicates that BTD-Lip was capable of staining much more lipid bodies than those stained by BODIPY. The hybrid dye showed therefore strong affinity with other lipid categories found in mammal cells, which BODIPY is unable to stain. Lipids found in mammal cells can be grouped in different categories such as fatty acyls, glycerolipids, grycerophospholipids, polyketides, sterol lipids, phenol lipids, sphingolipids, and saccharolipids, and these molecules have profound structural differences. It is likely that BTD-Lip is capable of associating with a more diverse lipid structures, and this feature provides the ability to stain other lipid bodies in the mammal cells, especially considering that the

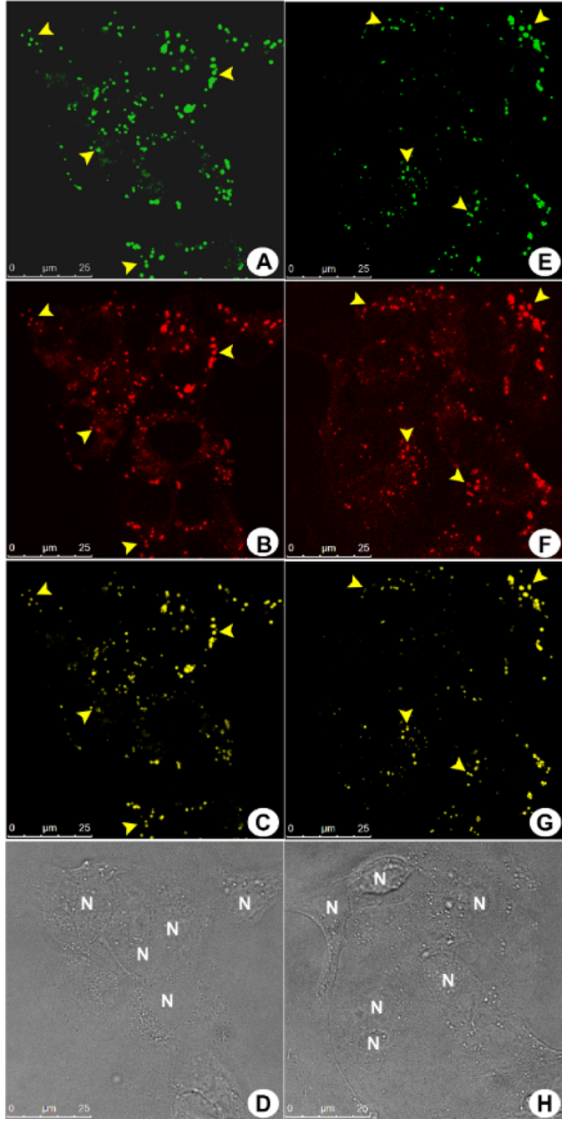

Figure 1. LDs selective staining using BTD-Lip with similar effectiveness in both live and fixed Caco- 2 cancer cells. (A-D) show the live samples, whereas $(\mathrm{E}-\mathrm{H})$ show fixed samples. $(\mathrm{A}, \mathrm{E})$ show the fluorescent aspect of LDs (arrows) using the commercially available BODIPY (green emission), whereas (B,F) LDs stained with BTD-Lip. $(\mathrm{D}, \mathrm{H})$ show the normal morphological aspects of the cells by phase contrast microscopy. (C,G) show the overlay between $(A, B)$ and $(\mathrm{E}, \mathrm{F})$, respectively, with yellow emission (red plus green), showing the match of the LDs using the commercial BODIPY and the developed BTD-Lip. The letter "N" indicates the cells' nuclei. Scale bar of $25 \mu \mathrm{m}$.

structure was designed to be lipophilic. The bright emission of BTD-Lip with neither photobleaching nor blinking allowed a precise quantification of the LD structures found in the cytosol of the cells (Figure 2).

In live cells, BTD-Lip stained ca. $32 \%$ more LD than BODIPY, whereas in fixed cells BTD-Lip stained ca. $40 \%$ more lipid structures when compared with the commercial dye. These results rendered BTD-Lip as a superior LD marker, and it can be used to follow the dynamics of lipid structures in live cells including other lipid-based structures (fatty acyls, glycerolipids, sphingolipids, and saccharolipids), a feature not possible using the widely used BODIPY. The water solubility of BTD-Lip was $2.5 \mathrm{mg} \mathrm{mL}^{-1}$ at room temperature, meaning that at the given concentration used at the cellular experiments (3 $\mu \mathrm{M})$, the dye was completely soluble, as expected. Its lipophilic character is in this sense the key toward lipid affinity and not its solubility.

BTD-Lip also had its capacity to be used associated with different dyes evaluated (Figure 3). In the experiment, BTDLip was tested alongside the commercially available DAPI (for nuclei staining and blue emission) and BODIPY (green emission). A bioorthogonal analysis was also performed to 


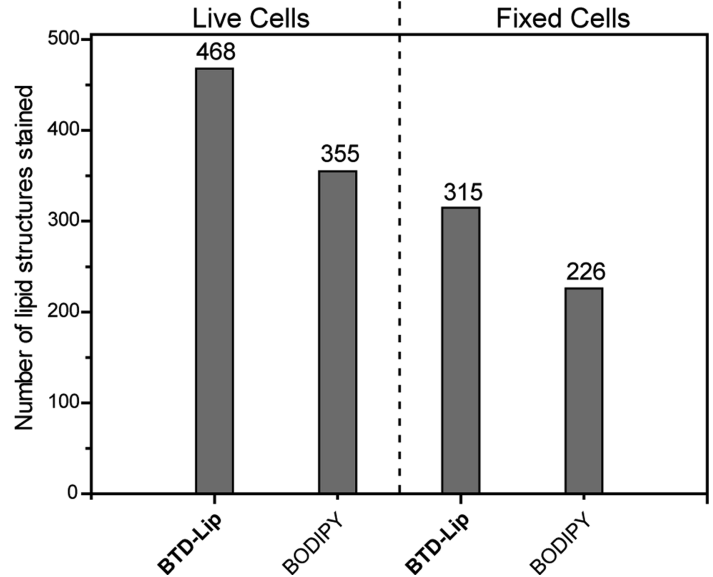

Figure 2. Lipid structure quantification from fluorescent profile of live and fixed Caco-2 cancer cells stained with BTD-Lip and with commercially available BODIPY.

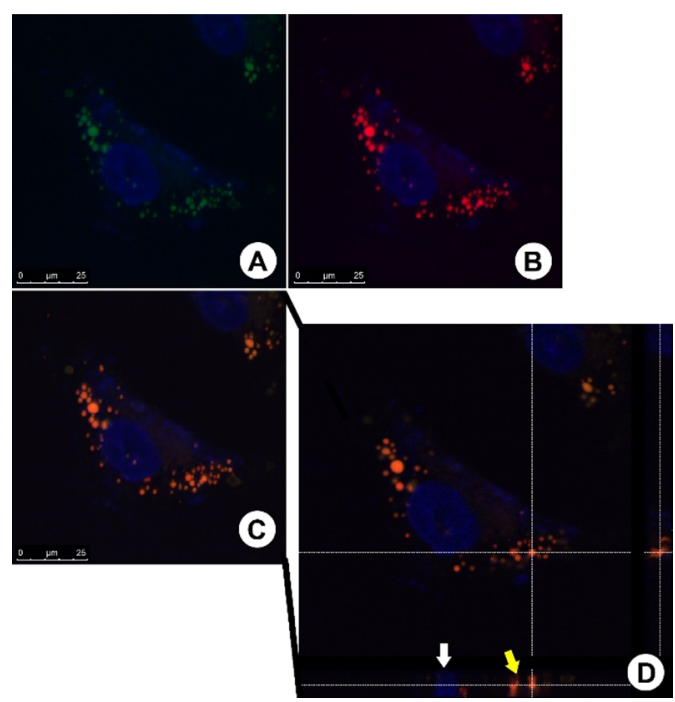

Figure 3. Fluorescent staining pattern and orthogonal analysis. (A,B) Fluorescent-stained cells with BODIPY and BTD-LIP, respectively. (C) Overlay of the two fluorescent signals. (D) Orthogonal analysis of the overlapped images. The white arrow is indicating a cell nucleus (stained with DAPI-blue emission) position in the orthogonal projection, and the yellow arrow is indicating the $\mathrm{LD}$ position. The same feature is visualized in the right side of the image (secondary orthogonal projection). The white dashed lines indicate the transversal planes from the cut. Scale bar of $25 \mu \mathrm{m}$.

ensure BTD-Lip was inside the cells rather than in their surface (Figure 3D). The orthogonal analyses afforded 50 images in the $Z$-axis. The images were staked and then a three-dimensional (3D) projection was performed with all of the 50 images. The $3 \mathrm{D}$ projection was transversally cut in two different points, that is, the inner area of the cut $3 \mathrm{D}$ projection from the horizontal and vertical orthogonal planes (see Experimental Section for more details).

The analyses confirmed that all spherical structures located in the cells' cytoplasm were positively stained by BODIPY (Figure 3A) and BTD-Lip (Figure 3B). This assay had established that these spherical structures are in fact LD. This assay also revealed that both fluorescent agents have the same homogeneous distribution inside of the lipid structures, producing therefore a fluorescent signal colocalized in the spatial fluorescent signal overlap (Figure 3C) as an orange (red + green) emission. The orthogonal analyses of the cells demonstrated that the spherical structures stained using BODIPY or BTD-Lip are truly located inside the cells' cytoplasm (Figure 3D).

Because of the high fluorescence intensity and wide distribution of the LD stained using the hybrid BTD-Lip, an analysis of distribution could also be realized and compared with the commercial BODIPY (Figure 4).
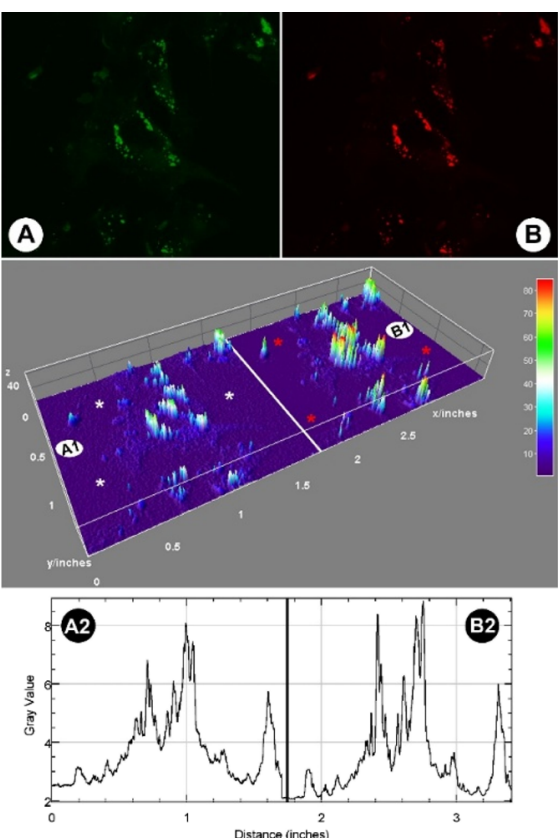

Figure 4. Fluorescence profile and intensity analyzes. (A,B) Caco-2 cells stained with BODIPY and BTD-Lip, respectively. (A1,B1) 3D intensity histogram projected over the fluorescence images and their respective fluorescence intensities scales shown in the right upper corner of the image. The white asterisks are indicating background staining, and the red asterisks show no background staining. (A2,B2) Two-dimensional (2D) histogram representation for fluorescence intensities of both dyes.

The analyses showed interesting results. Figure 4A,B shows several lipid bodies dispersed in the peripheral region in the cytoplasm. The fluorescence intensity analyses in the same image provide a remarkable evidence of higher intensity of the fluorescent signal produced by BTD-Lip against the low fluorescence intensity produced by the commercial dye (Figure 4, panels A1 and B1). These results are in agreement with the previous observation (Figure 2). The intensity analyses also showed a medium staining in the background produced by using BODIPY. The stained background is represented by the granularity in the frame outside the cells area (Figure 4, panel B1) indicated by white asterisks. No background staining was contrariwise produced by using BTD-Lip (Figure 4, panel B2), as noted in the red asterisks. In this case, no granularity in the frame outside the cell area could be noted, indicating the higher efficiency of the designed hybrid probe. The intensity result was also plotted in a 2D histogram (Figure 4, panels B1 and B2) to show clear-cut differences between the fluorescence intensities of both compounds.

To gain insights on the generality of BTD-Lip as the selective LD marker, additional cancer cell lineages have been 


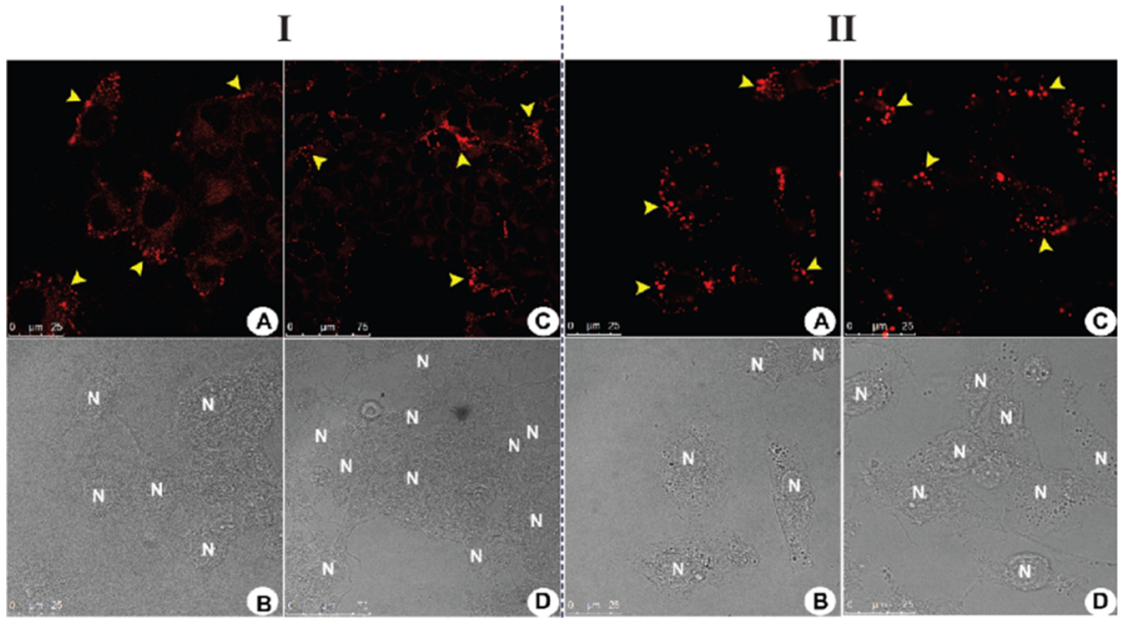

Figure 5. (I) MCF-7 cancer cells. (II) MDA-MB-231 cancer cells. (A,B) Live cells, whereas (C,D) fixed samples. (B,D) Normal morphological aspects of the samples by phase-contrast microscopy. Yellow arrow heads denote the spherical structure widely distributed within the cytoplasm. No staining structures were found inside of the cellular nuclei. The letter " $N$ " corresponds to cells' nuclei regions. Reference scale bar of $25 \mu$ m.

tested (Figure 5), that are MCF-7 (breast cancer cells) and MDA-MB-231 (invasive human breast adenocarcinoma cells).

Encouraged by the superior results that we obtained using BTD-Lip, we tested its ability against the complex live model, C. elegans (Figure 6).

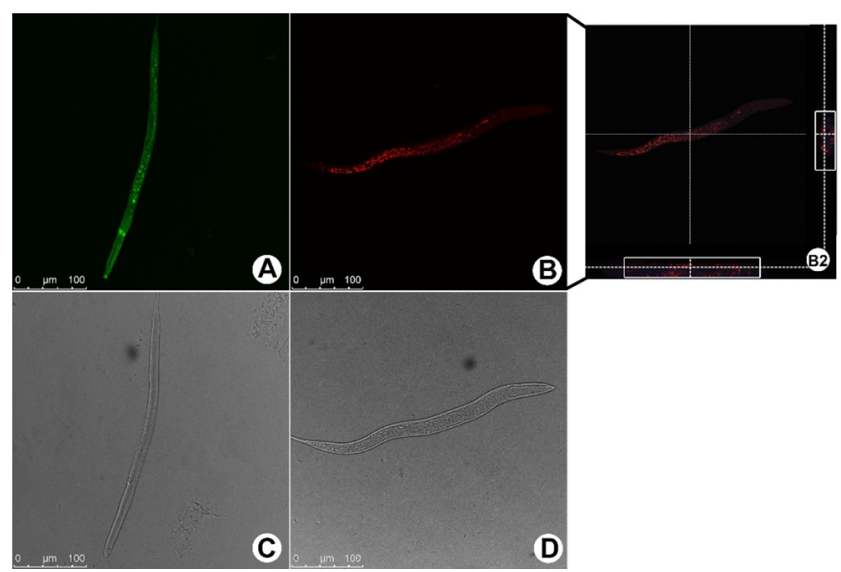

Figure 6. Staining pattern and orthogonal analyses of C. elegans using the commercial BODIPY (panel (A) green emission) and the hybrid designed probe BTD-Lip (panel (B), red emission). (B) Remarkable specificity in the worm stained with BTD-Lip in the region known to be rich in lipid structures, whereas no selectivity is noted using the commercial dye (A) which is noted along the whole worm. (B2) Orthogonal analyses of C. elegans stained with BTD-Lip, and the lipids bodies are located inside in the worm body as may be observed in the boxes at bottom and right sides. (C,D) Normal morphological aspects of the samples by phase-contrast microscopy Reference scale bar of $100 \mu \mathrm{m}$.

The BODIPY staining pattern observed in the C. elegans shows the lipids bodies positively stained but displaying an unspecific staining background distributed all along worm body (Figure 6A). The fluorescent pattern observed in the nematodes stained with BTD-Lip, however, was much more intense and highly specific (Figure 6B). No background staining could be observed in the worm. An accurate lipid body staining showing several spherical structures dispersed inside of all nematode body with a slight accumulation in the posterior region of the worm body (Figure 6B) was clear-cut using the hybrid designed dye. The inner position of the lipid bodies stained with BTD-Lip are clearly identified in the orthogonal projection (Figure 6B2). These regions are known to be lipid-rich, therefore confirming the high affinity of BTDLip for lipid structures even inside live complex models.

Finally, results obtained herein were reproduced once more using a stock solution stored for 30 months. No degradation was noted in the structure of BTD-Lip standing at an ambient temperature at the laboratory facility. The commercial dye, as described by the manufacturer, may be kept at $-20{ }^{\circ} \mathrm{C}$ and cannot be frozen again.

\section{CONCLUSIONS}

In summary, a new fluorescent hybrid BTD-coumarin dye (BTD-Lip) was successfully synthesized, characterized, and tested as a superior marker for lipid structures, especially LDs. The new compound proved to be chemically stable and photostable and does not undergo degradation from excited states.

Using the designed probe, a considerable larger number of lipid structures could be stained when compared with the commercial dye independent of whether live or fixed cells were used. When a complex model (C. elegans) was tested, superior results were once more obtained, and the designed probe stained only the region known to be lipid-rich, whereas the commercial dye showed no selectivity. The knowledge gained by this work may be the basis for the development of new hybrid BTD-based structures for selective cellular staining and for complex live models such as $C$. elegans. The designed structure indeed prevailed where commercial dyes fell short.

\section{EXPERIMENTAL SECTION}

General. The nuclear magnetic resonance spectra of hydrogen $\left({ }^{1} \mathrm{H}\right.$ NMR $)$ and carbon $\left({ }^{13} \mathrm{C}\right.$ NMR $)$ were obtained with a $600 \mathrm{MHz}$ NMR instrument. Chemical shifts were expressed in parts per million and referenced by the signals of TMS or of the residual hydrogen atoms of the deuterated solvent $\left(\mathrm{CDCl}_{3}\right)$ depending on the case, as indicated in the legends.

Synthetic Procedure. Synthesis of the monosubstituted BTD derivative: 4,7-dibromo-2,1,3-benzothiadiazole (0.3 
mmol, $100 \mathrm{mg}$ ), 4-methoxyphenylboronic acid (0.3 mmol, 50 $\mathrm{mg}$ ), $5 \mathrm{~mol} \%$ of $\mathrm{Pd}(\mathrm{OAc})_{2}$ (ca. $4 \mathrm{mg}$ ), $10 \mathrm{~mol} \%$ of $\mathrm{PPh}_{3}$ (ca. 8 $\mathrm{mg}), 100 \mathrm{mg}$ of a solution of $\mathrm{Na}_{2} \mathrm{CO}_{3}(2 \mathrm{M})$, and $5 \mathrm{~mL}$ of toluene are added in a sealed Schlenk tube for $12 \mathrm{~h}$ at $100{ }^{\circ} \mathrm{C}$. The crude mixture was filtered through celite and chromatographed using silica (230 mesh) eluted using hexane/ethyl acetate $(3: 1 \mathrm{v} / \mathrm{v})$. Yield: $60 \%$. Considering this to be a known compound, it was used in the second step without any characterization.

Synthesis of BTD-Lip (7-((7-(4-methoxyphenyl)benzo[c]$[1,2,5]$ thiadiazol-4-yl)amino)-4-methyl-2 $H$-chromen-2-one): The monosubstituted BTD $(0.5 \mathrm{mmol}, 160 \mathrm{mg})$, 7-amino-4methylcoumarin $(0.5 \mathrm{mmol}, 88 \mathrm{mg}), 5 \mathrm{~mol} \%$ of $\mathrm{Pd}(\mathrm{OAc})_{2}$ (ca. $5 \mathrm{mg}$ ), $10 \mathrm{~mol} \% \mathrm{PPh}_{3}$ (ca. $13 \mathrm{mg}$ ), $200 \mathrm{~mol} \%$ of ${ }^{t} \mathrm{BuOK}(28$ $\mathrm{mg})$, and anhydrous toluene $(5 \mathrm{~mL})$ were added to a sealed Schlenk tube for $72 \mathrm{~h}$ at $100{ }^{\circ} \mathrm{C}$. The crude mixture was filtered through celite and chromatographed using silica (230 mesh), eluted using hexane/ethyl acetate $(3: 1 \mathrm{v} / \mathrm{v})$. Yield: $30 \%$. Melting point: $182{ }^{\circ} \mathrm{C} .{ }^{1} \mathrm{H}$ NMR $\left(600 \mathrm{MHz}, \mathrm{CDCl}_{3}\right) \delta$ ppm: 7.89 (d, $9.17 \mathrm{~Hz}, 2 \mathrm{H}), 7.64(\mathrm{~d}, 7.70 \mathrm{~Hz}, 1 \mathrm{H}) 7.57(\mathrm{~d}, 8.80 \mathrm{~Hz}$, $1 \mathrm{H}), 7.49(\mathrm{~d}, 7.70 \mathrm{~Hz}, 1 \mathrm{H}) 7.45(\mathrm{br}, 1 \mathrm{H}) 7.36(\mathrm{~d}, 2.20 \mathrm{~Hz}, 1 \mathrm{H})$ 7.18 (dd, 9.17; $2.36 \mathrm{~Hz}, 1 \mathrm{H}) 7.08(\mathrm{~d}, 8.80 \mathrm{~Hz}, 2 \mathrm{H}) 6.17(\mathrm{~s}, 1 \mathrm{H})$ 3.89 (s, 3H), $2.44(\mathrm{~s}, 3 \mathrm{H}) ;{ }^{13} \mathrm{C}$ NMR (150 MHz, $\left.\mathrm{CDCl}_{3}\right) \delta$ ppm: 174.0, 161.2, 159.4, 155.3, 154.0, 152.3, 149.1, 144.6, $132.1,129.9,128.3,126.3,125.8,114.8,114.2,112.2,109.3$, 104.4, 55.4, 18.6. Anal. Calcd for $\mathrm{C}_{23} \mathrm{H}_{17} \mathrm{~N}_{3} \mathrm{O}_{3} \mathrm{~S}$ : C, 66.49; $\mathrm{H}$, 4.12; N, 10.11. Found: C, 66.58; H, 4.20; N, 10.22. IR (KBr, $\left.\mathrm{cm}^{-1}\right): 3319,3094,3061,2917,1718,1615,1530,1383$. Water solubility $\left(25^{\circ} \mathrm{C}\right): 2.5 \mathrm{mg} \mathrm{mL}^{-1}$.

Cellular Staining Procedures. Cell Cultures. MCF-7 (human breast adenocarcinoma cell), MDA-MB-231 (human breast invasive adenocarcinoma cell), and Caco-2 (human epithelial colorectal adenocarcinoma) cells were used. All cell lineages were maintained in an appropriate culture medium as recommended by the American Type Culture Collection, supplemented with $10 \%$ fetal bovine serum along with $100 \mathrm{IU} /$ $\mathrm{mL}$ penicillin and $100 \mu \mathrm{g} \mathrm{mL} \mathrm{m}^{-1}$ streptomycin at $37{ }^{\circ} \mathrm{C}$ in $5 \%$ $\mathrm{CO}_{2}$ atmosphere.

Fluorescent Assays. Cells were seeded on $13 \mathrm{~mm}$ round glass coverslips on the bottom of a 24-well plate, allowed to adhere overnight, and washed three times with a serum-free medium for the removal of nonadherent cells. After reaching confluence, the cells were separated into two samples (live samples and fixed samples). The live samples were incubated for $30 \mathrm{~min}$ in $3 \mu \mathrm{M}$ solution of BTD-Lip at $37{ }^{\circ} \mathrm{C}$. These samples were washed three times with phosphate-buffered saline (PBS) $1 \times(\mathrm{pH} \mathrm{7.4)}$ at room temperature and fixed in $3.7 \%$ formaldehyde for $30 \mathrm{~min}$. The samples were washed again thrice in PBS $1 \times(\mathrm{pH} \mathrm{7.4)}$ at room temperature, and the coverslips were mounted over glass slides using ProLong Gold Antifade (Invitrogen, OR, USA) according to the manufacturer's recommendations. The fixed samples were first washed thrice in PBS $1 \times(\mathrm{pH} 7.4)$ and then fixed in $3.7 \%$ formaldehyde for $30 \mathrm{~min}$. After a fixative procedure, the samples were washed thrice in PBS $1 \times(\mathrm{pH} 7.4)$ at room temperature and incubated for $30 \mathrm{~min}$ in $3 \mu \mathrm{M}$ solution of BTD-Lip at $37^{\circ} \mathrm{C}$. The samples were washed thrice in PBS $1 \times(\mathrm{pH} 7.4)$ at room temperature, and the coverslips were mounted over glass slides using ProLong Gold Antifade (Invitrogen, OR, USA) according to the manufacturer's recommendations. The negative control was performed by the incubation of the samples using $0.1 \%$ dimethyl sulfoxide (DMSO), which was the diluent used. The samples were analyzed using a Leica Confocal Microscopy TCS
SP5 and excited using $488 \mathrm{~nm}$ wavelength laser emission. All assays were performed in triplicates, and it was performed thrice for each cell sample under the experimental condition.

Neutral Lipid Staining with Commercial BODIPY. The staining procedures with BODIPY were performed with the same cell groups (live and fixed cells) as described above. Briefly, the cells samples were incubated with BODIPY using DMSO solution $(12 \mu \mathrm{M})$ during $30 \mathrm{~min}$ at room temperature. After the incubation of samples, the cells were washed thrice in PBS, and the samples were mounted over glass slides by using antifade agent Prolong Gold (Invitrogen, OR, USA), according to the manufacture's recommendations. The samples were analyzed using a Leica Confocal Microscopy TCS SP5 and excited using $488 \mathrm{~nm}$ wavelength laser emission. All assays were performed in triplicate, and it was performed thrice for each cell sample and experimental condition.

Lipid Structures Quantification. Alipid quantification by using ImageJ free software was performed. The images obtained from BODIPY- and BTD-Lip-stained samples were adjusted to the same color threshold values. Also, the images were treated by a level of adjustment to maximize the background removal. The particle analysis tool with the range of circularity setup of 0.25-1.00 was applied. The in situ option shows that overlay outlines were selected in the software to be a possible visual inspection of the automatic selection. The adjustment described above was able to include all lipids structures from the images.

Cell Viability Assay. The viability tests were performed with MCF-7 (human breast adenocarcinoma cell line), MDA-MB231 (human breast Invasive ductal carcinoma), and Caco-2 (human colon epithelial cancer cell line). The cells were incubated with the BTD-Lip at $100 \mu \mathrm{M}$ for $24 \mathrm{~h}$ and analyzed by standard MTT assays, following the manufacturer's recommendations (R\&D System Inc, MN, USA). Briefly, $3 \times$ $10^{3}$ of each cell line were seeded in a 96 well plate and maintained overnight a $37{ }^{\circ} \mathrm{C}$. These sample tests were incubated in $100 \mu \mathrm{M}$ of BTD-Lip solution for $24 \mathrm{~h}$. The cells incubated with the solvent agent DMSO (down to $0.1 \%$ ) at the same conditions and maintained only in the culture medium were used as positive and negative controls, respectively. The samples were incubated with $150 \mu \mathrm{L}$ of MTT solution $(0.5 \mathrm{mg}$ $\left.\mathrm{mL}^{-1}\right)$ in the cell culture medium for $4 \mathrm{~h}$ in the dark at $37^{\circ} \mathrm{C}$. MTT is reduced by metabolically active cells to insoluble purple formazan dye crystals that were accumulated inside the cells' cytoplasm. The MTT solution was removed and $200 \mu \mathrm{L}$ of DMSO was added in all samples to solubilize the formazan dye crystals. The plate was read in spectrophotometer Spectramax M5 (Molecular Devices, CA, USA), and the optimal wavelength for absorbance was $570 \mathrm{~nm}$. The MTT assay was performed in triplicate and also made three independent assays. The cell viability inhibition was determined by evaluation of MTT result obtained for test samples compared with the control samples in the same conditions, following the expression \{survival $\%=[$ (tested sample blank $) /($ control sample - blank $)] \times 100\}$.

C. elegans Maintenance and Staining Procedures. The nematodes were maintained in the nematode growth medium (NGM agar medium) inoculated with Escherichia coli (NA22 strain) in petri dishes. The worms were seeded and kept at 22 ${ }^{\circ} \mathrm{C}$ for 30 days. The M2 medium was added to the petri dishes, and the M2 medium and worms were collected and transferred to plastic tubes $(1.5 \mathrm{~mL})$ and centrifuged at $2348 \mathrm{~g}$ for $1 \mathrm{~min}$. The supernatant was discarded, and this process was repeated 
twice. The worms were incubated in $4 \%$ paraformaldehyde for $15 \mathrm{~min}$ and the samples were submitted to freeze (in $\mathrm{N}_{2}$ ) and thaw (in water at room temperature). The samples were washed thrice in M2 medium and incubated for $2 \mathrm{~h}$ with BTDLip $(3 \mu \mathrm{M})$ or in BODIPY $(12 \mu \mathrm{M})$. The samples were washed thrice in M2 medium and put over glass slide containing poly-Llysine at $0.1 \%$. The samples were mounted by using Prolong Gold antifade agent. The images acquisitions were performed by using the Leica TSC-SP5 confocal microscope. The assays involving $C$. elegans were performed in triplicate, and the experiments were repeated thrice.

Orthogonal Analysis from Cell Samples and C. elegans. Samples were used to produce 50 images in the $Z$-axis. The images were stacked, and a $3 \mathrm{D}$ projection with all 50 images was performed. The $3 \mathrm{D}$ projection was transversally cut in two different points. The software module of the orthogonal analysis (LAS-AF, Leica) created two new images, which show the inner area of the cut $3 \mathrm{D}$ projection from the horizontal and vertical orthogonal cut planes.

Photostability Assay. A $10 \mu \mathrm{M}$ solution of BTD-Lip was placed in a 96-well plate. The fluorescence measurements were recorded every $5 \mathrm{~min}$ for a total period of $6 \mathrm{~h}(\mathrm{Ex} / \mathrm{Em}=480 /$ $610 / 695)$ under a tungsten halogen light source constant irradiation. The values were represented as means $(n=3)$ and fitted to a nonlinear regression one-phase exponential decay using GraphPad Prism 5.0 for Windows, GraphPad Software, (San Diego CA, USA).

\section{ASSOCIATED CONTENT}

\section{S Supporting Information}

The Supporting Information is available free of charge on the ACS Publications website at DOI: 10.1021/acsomega.8b00434.

UV-vis, fluorescent emission, and Stokes shift versus ETN values; cell viability assays; $1 \mathrm{H} \mathrm{NMR} \mathrm{(CDCl3,} 600$ $\mathrm{MHz})$ of BTD-Lip; 13C NMR (CDCl3, $150 \mathrm{MHz})$ of BTD-Lip; fluorescence intensity of BTD-Lip; and photostability assay (PDF)

\section{AUTHOR INFORMATION}

\section{Corresponding Author}

*E-mail: brenno.ipi@gmail.com (B.A.D.N.).

ORCID ${ }^{\circ}$

Brenno A. D. Neto: 0000-0003-3783-9283

Notes

The authors declare no competing financial interest.

\section{ACKNOWLEDGMENTS}

This work has been supported by CAPES, CNPq, FINATEC, FAPDF, FAPESP, and DPP-UnB. B.A.D.N. also thanks INCTTranscend group. LNLS is acknowledged for the use of the facilities.

\section{REFERENCES}

(1) Chan, J.; Dodani, S. C.; Chang, C. J. Reaction-based smallmolecule fluorescent probes for chemoselective bioimaging. Nat. Chem. 2012, 4, 973-984.

(2) Nadler, A.; Schultz, C. The Power of Fluorogenic Probes. Angew. Chem., Int. Ed. 2013, 52, 2408-2410.

(3) Lavis, L. D.; Raines, R. T. Bright Building Blocks for Chemical Biology. ACS Chem. Biol. 2014, 9, 855-866.
(4) Boldyrev, I. A.; Zhai, X.; Momsen, M. M.; Brockman, H. L.; Brown, R. E.; Molotkovsky, J. G. New BODIPY lipid probes for fluorescence studies of membranes. J. Lipid Res. 2007, 48, 1518-1532.

(5) Lavis, L. D.; Raines, R. T. Bright Ideas for Chemical Biology. ACS Chem. Biol. 2008, 3, 142-155.

(6) Rumin, J.; Bonnefond, H.; Saint-Jean, B.; Rouxel, C.; Sciandra, A.; Bernard, O.; Cadoret, J.-P.; Bougaran, G. The use of fluorescent Nile red and BODIPY for lipid measurement in microalgae. Biotechnol. Biofuels 2015, 8, 42.

(7) Yang, H.-J.; Hsu, C.-L.; Yang, J.-Y.; Yang, W. Y. Monodansylpentane as a Blue-Fluorescent Lipid-Droplet Marker for MultiColor Live-Cell Imaging. PLoS One 2012, 7, No. e32693.

(8) Bruegger, B. Lipidomics: Analysis of the Lipid Composition of Cells and Subcellular Organelles by Electrospray Ionization Mass Spectrometry. In Annual Review of Biochemistry; Kornberg, R. D., Ed.; Annual Reviews, 2014; Vol. 83, pp 79-98.

(9) Neef, A. B.; Schultz, C. Selective Fluorescence Labeling of Lipids in Living Cells. Angew. Chem., Int. Ed. 2009, 48, 1498-1500.

(10) Hulme, S. E.; Whitesides, G. M. Chemistry and the Worm: Caenorhabditis elegans as a Platform for Integrating Chemical and Biological Research. Angew. Chem., Int. Ed. 2011, 50, 4774-4807.

(11) Hellerer, T.; Axaeng, C.; Brackmann, C.; Hillertz, P.; Pilon, M.; Enejder, A. Monitoring of lipid storage in Caenorhabditis elegans using coherent anti-Stokes Raman scattering (CARS) microscopy. Proc. Natl. Acad. Sci. U.S.A. 2007, 104, 14658-14663.

(12) Ashrafi, K.; Chang, F. Y.; Watts, J. L.; Fraser, A. G.; Kamath, R. S.; Ahringer, J.; Ruvkun, G. Genome-wide RNAi analysis of Caenorhabditis elegans fat regulatory genes. Nature 2003, 421, 268272.

(13) Goel, A.; Sharma, A.; Kathuria, M.; Bhattacharjee, A.; Verma, A.; Mishra, P. R.; Nazir, A.; Mitra, K. New Fluoranthene FLUN-550 as a Fluorescent Probe for Selective Staining and Quantification of Intracellular Lipid Droplets. Org. Lett. 2014, 16, 756-759.

(14) Kim, E.; Lee, S.; Park, S. B. A Seoul-Fluor-based bioprobe for lipid droplets and its application in image-based high throughput screening. Chem. Commun. 2012, 48, 2331-2333.

(15) Lee, J. H.; So, J.-H.; Jeon, J. H.; Choi, E. B.; Lee, Y.-R.; Chang, Y.-T.; Kim, C.-H.; Bae, M. A.; Ahn, J. H. Synthesis of a new fluorescent small molecule probe and its use for in vivo lipid imaging. Chem. Commun. 2011, 47, 7500-7502.

(16) Safari, J.; Gandomi-Ravandi, S.; Ashiri, S. Organosilane sulfonated graphene oxide in the Biginelli and Biginelli-like reactions. New J. Chem. 2016, 40, 512-520.

(17) Wang, H.; Guo, L. E.; Li, X. M.; Zhang, L. M.; Xu, Q. L.; Wu, G. F.; Zhou, Y.; Zhang, J. F. Coumarin-based turn-on fluorescence probes for highly selective detection of $\mathrm{Pi}$ in cell culture and Caenorhabditis elegans. Dyes Pigm. 2015, 120, 293-298.

(18) Yen, K.; Le, T. T.; Bansal, A.; Narasimhan, S. D.; Cheng, J.-X.; Tissenbaum, H. A. A Comparative Study of Fat Storage Quantitation in Nematode Caenorhabditis elegans Using Label and Label-Free Methods. PLoS One 2010, 5, No. e12810.

(19) Cooper, A. F.; Van gundy, S. D. Metabolism of glycogen and neutral lipids by aphelenchus-avenae and caenorhabditis-sp in aerobic, microaerobic, and anaerobic environments. J. Nematol. 1970, 2, 305315.

(20) Neto, B. A. D.; Carvalho, P. H. P. R.; Correa, J. R. Benzothiadiazole Derivatives as Fluorescence Imaging Probes: Beyond Classical Scaffolds. Acc. Chem. Res. 2015, 48, 1560-1569.

(21) Jiang, Q.; Zhang, Z.; Lu, J.; Huang, Y.; Lu, Z.; Tan, Y.; Jiang, Q. A Novel Nitro-Substituted Benzothiadiazole as Fluorescent Probe for Tumor Cells under Hypoxic Condition. Bioorg. Med. Chem. 2013, 21, $7735-7741$.

(22) Garcia, L.; Lazzaretti, M.; Diguet, A.; Mussi, F.; Bisceglie, F.; Xie, J.; Pelosi, G.; Buschini, A.; Baigl, D.; Policar, C. An Intrinsically Fluorescent Glycoligand for Direct Imaging of Ligand Trafficking in Artificial and Living Cell Systems. New J. Chem. 2013, 37, 3030-3034.

(23) Tian, Y.; Wu, W.-C.; Chen, C.-Y.; Jang, S.-H.; Zhang, M.; Strovas, T.; Anderson, J.; Cookson, B.; Li, Y.; Meldrum, D.; Chen, W.C.; Jeni, A. K.-Y. Utilization of Micelles Formed from Poly(ethylene 
glycol)-Block-Poly(epsilon-Caprolactone) Block Copolymers as Nanocarriers to Enable Hydrophobic Red Two-Photon Absorbing Emitters for Cells Imaging. J. Biomed. Mater. Res., Part A 2010, 93, $1068-1079$.

(24) Yao, S.; Kim, B.; Yue, X.; Gomez, M. Y. C.; Bondar, M. V.; Belfield, K. D. Synthesis of Near-Infrared Fluorescent Two-PhotonAbsorbing Fluorenyl Benzothiadiazole and Benzoselenadiazole Derivatives. ACS Omega 2016, 1, 1149-1156.

(25) Yang, D.; Wang, H.; Sun, C.; Zhao, H.; Hu, K.; Qin, W.; Ma, R.; Yin, F.; Qin, X.; Zhang, Q.; Liang, Y.; Li, Z. Development of a high quantum yield dye for tumour imaging. Chem. Sci. 2017, 8, 63226326.

(26) Dyrager, C.; Vieira, R. P.; Nystrom, S.; Nilsson, K. P. R.; Storr, T. Synthesis and evaluation of benzothiazole-triazole and benzothiadiazole-triazole scaffolds as potential molecular probes for amyloidbeta aggregation. New J. Chem. 2017, 41, 1566-1573.

(27) Chen, C.; Hua, Y.; Hu, Y.; Fang, Y.; Ji, S.; Yang, Z.; Ou, C.; Kong, D.; Ding, D. Far-red/near-infrared fluorescence light-up probes for specific in vitro and in vivo imaging of a tumour-related protein. Sci. Rep. 2016, 6, 23190.

(28) Mota, A. A. R.; Carvalho, P. H. P. R.; Guido, B. C.; de Oliveira, H. C. B.; Soares, T. A.; Corrêa, J. R.; Neto, B. A. D. Bioimaging, Cellular Uptake and Dynamics in Living Cells of a Lipophilic Fluorescent Benzothiadiazole at Low Temperature $\left(4{ }^{\circ} \mathrm{C}\right)$. Chem. Sci. 2014, 5, 3995-4003.

(29) Appelqvist, H.; Stranius, K.; Börjesson, K.; Nilsson, K. P. R.; Dyrager, C. Specific Imaging of Intracellular Lipid Droplets Using a Benzothiadiazole Derivative with Solvatochromic Properties. Bioconjugate Chem. 2017, 28, 1363-1370.

(30) Carvalho, P. H. P. R; Correa, J. R.; Guido, B. C.; Gatto, C. C.; De Oliveira, H. C. B.; Soares, T. A.; Neto, B. A. D. Designed Benzothiadiazole Fluorophores for Selective Mitochondrial Imaging and Dynamics. Chem.-Eur. J. 2014, 20, 15360-15374.

(31) Dias, G. G.; Rodrigues, B. L.; Resende, J. M.; Calado, H. D. R.; de Simone, C. A.; Silva, V. H. C.; Neto, B. A. D.; Goulart, M. O. F.; Ferreira, F. R.; Meira, A. S.; Pessoa, C.; Correa, J. R.; da Silva Júnior, E. $\mathrm{N}$. Selective endocytic trafficking in live cells with fluorescent naphthoxazoles and their boron complexes. Chem. Commun. 2015, 51, 9141-9144.

(32) Diniz, J. R.; Correa, J. R.; Moreira, D. D. A.; Fontenele, R. S.; de Oliveira, A. L.; Abdelnur, P. V.; Dutra, J. D. L.; Freire, R. O.; Rodrigues, M. O.; Neto, B. A. D. Water-Soluble $\mathrm{Tb}^{3+}$ and $\mathrm{Eu}^{3+}$ Complexes with Ionophilic (Ionically Tagged) Ligands as Fluorescence Imaging Probes. Inorg. Chem. 2013, 52, 10199-10205.

(33) Neto, B. A. D.; Lapis, A. A. M.; da Silva Júnior, E. N.; Dupont, J. 2,1,3-Benzothiadiazole and Derivatives: Synthesis, Properties, Reactions, and Applications in Light Technology of Small Molecules. Eur. J. Org. Chem. 2013, 2013, 228-255.

(34) Neto, B. A. D.; Corrêa, J. R.; Silva, R. G. Selective Mitochondrial Staining with Small Fluorescent Probes: Importance, Design, Synthesis, Challenges and Trends for new Markers. RSC Adv. 2013, 3, 5291-5301.

(35) Xiong, K.; Huo, F.; Yin, C.; Yang, Y.; Chao, J.; Zhang, Y.; Xu, M. A off-on green fluorescent chemosensor for cyanide based on a hybrid coumarin-hemicyanine dye and its bioimaging. Sens. Actuators, B 2015, $220,822-828$.

(36) Wu, A.; Kolanowski, J. L.; Boumelhem, B. B.; Yang, K.; Lee, R.; Kaur, A.; Fraser, S. T.; New, E. J.; Rendina, L. M. A CarboraneContaining Fluorophore as a Stain of Cellular Lipid Droplets. Chem.Asian J. 2017, 12, 1704-1708.

(37) Hu, L.; Wang, H.; Xu, X.; Zhang, M.; Tian, X.; Wu, J.; Zhou, H.; Yang, J.; Tian, Y. A small-molecule with large two-photon action crosssection serves as the membrane-permeable probe for live cells imaging and bacteria viability. Sens. Actuators, B 2017, 241, 1082-1089.

(38) Kraljević, T. G.; Harej, A.; Sedić, M.; Pavelić, S. K.; Stepanić, V.; Drenjančević, D.; Talapko, J.; Raić-Malić, S. Synthesis, in vitro anticancer and antibacterial activities and in silico studies of new 4substituted 1,2,3-triazole-coumarin hybrids. Eur. J. Med. Chem. 2016, 124, 794-808.
(39) Reichardt, C. Solvatochromic dyes as solvent polarity indicators. Chem. Rev. 1994, 94, 2319-2358.

(40) Ravi, M.; Soujanya, T.; Samanta, A.; Radhakrishnan, T. P. Excited-state dipole-moments of some coumarin dyes from a solvatochromic method using the solvent polarity parameter, $\mathrm{E}(\mathrm{N})$ (T). J. Chem. Soc., Faraday Trans. 1995, 91, 2739-2742.

(41) Takadate, A.; Masuda, T.; Murata, C.; Tanaka, T.; Irikura, M.; Goya, S. Fluorescence Characteristics of Methoxycoumarins as Novel Fluorophores. Anal. Sci. 1995, 11, 97-101.

(42) Ishow, E.; Clavier, G.; Miomandre, F.; Rebarz, M.; Buntinx, G.; Poizat, O. Comprehensive investigation of the excited-state dynamics of push-pull triphenylamine dyes as models for photonic applications. Phys. Chem. Chem. Phys. 2013, 15, 13922-13939.

(43) Gautam, P.; Yu, C. P.; Zhang, G.; Hillier, V. E.; Chan, J. M. W. Pulling with the Pentafluorosulfanyl Acceptor in Push Pull Dyes. J. Org. Chem. 2017, 82, 11008-11020.

(44) Lee, K.; Kunjappu, J.; Jockusch, S.; Turro, N. J.; Widerschpan, T.; Zhou, J. M.; Smith, B. W.; Zimmerman, P.; Conley, W. Amplification of the index of refraction of aqueous immersion fluids by ionic surfactants. In Advances in Resist Technology and Processing XXII, Pt 1 and 2; Sturtevant, J. L., Ed.; Proceedings of the Society of Photo-Optical Instrumentation Engineers (SPIE), 2005; Vol. 5753, pp 537-553. 